\title{
Analogía y sublimación: una respuesta al problema del pasaje del silencio a la palabra en la filosofia de Merleau-Ponty
}

\author{
Martín Buceta \\ UCA/CONICET
}

\begin{abstract}
Resumen: El presente artículo tiene como propósito explicar cómo puede darse el pasaje del mundo de la percepción al mundo de la expresión en la filosofia de M. Merleau-Ponty. Para llevar a cabo este objetivo intentaremos exponer las dos vías que nos permitirán esbozar una respuesta al problema y que pueden advertirse en la obra del autor. La primera de ellas consiste en establecer la analogía que existe entre la estructuración del sentido perceptivo y el del lenguaje, en ambos se dará de modo diacrítico. La segunda es aquella de la sublimación que posibilita la palabra (parole) como elemento carnal que es parte del mundo visible pero prolonga en lo invisible. Finalmente apelaremos a la noción de fe expresiva para sustentar la relación entre palabra y realidad.
\end{abstract}

Palabras clave: pasaje; palabra; carne; analogía; sublimación

\begin{abstract}
Analogy and Sublimation: an Answer to the Problem of the Passage from Silence to Word in the Philosophy of Merleau-Ponty". The purpose of this article is to explain how the passage from the world of perception to the world of expression can occur in the philosophy of M. Merleau-Ponty. To carry out this objective we will try to expose the two ways that will allow us to outline an answer to the problem, both of which can be noticed in the work of the author. The first of these is to establish the analogy that exists between the structuring of the perceptive sense and of language, in both it will happen in a diacritical way. The second is that of sublimation, which enables the word (parole) as a carnal element that is part of the visible world but extends into the invisible. Finally, we will appeal to the notion of expressive faith to sustain the relationship between word and reality.

Keywords: passage; word; flesh (chair); analogy; sublimation
\end{abstract}




\section{Introducción}

Uno de los problemas que permanece irresuelto en la Fenomenología de la percepción es la explicitación de la relación entre el ser percibido y el lenguaje, es decir, la ausencia de una explicación "[d]el problema del pasaje

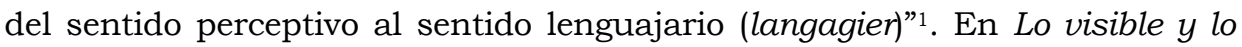
invisible, Merleau-Ponty había emprendido un proyecto que tenía por objetivo elaborar una nueva ontología. Esta aspiración se venía gestando, al modo de una "sintesis pasiva", desde los cursos dictados en el Collège de France. En aquellos cursos el autor anunciaba que hasta ese momento habia realizado una aproximación al mundo percibido organizada en función de conceptos clásicos como percepción, conciencia, síntesis, etcétera ${ }^{2}$. Sin embargo, estos le parecian insuficientes porque tal terminología le imponía limites a los análisis que pretendia llevar adelante -aquellos que querían dar cuenta del pasaje del mundo sensible al mundo de la expresión-y, por lo tanto, buscaría establecer un nuevo campo de conceptos para dicho propósito. La nueva ontología iniciada en los Cursos y continuada en Lo visible y lo invisible-aunque inconclusa a causa de la muerte del autor- puso en juego elementos clave para pensar el pasaje de un logos a otro, es decir, del silencio a la palabra. Estos fueron las nociones de carne (chair), institución, conciencia perceptiva, dimensión, etcétera.

Hacia el final de su obra, Merleau-Ponty esbozará una vía de superación de dicho problema, de modo que la noción de carne (chair) se posicionará como el pivote necesario para explicar la articulación entre una dimensión y otra. Este elemento se perfila como el concepto central para entender en conjunto la unidad del sentido perceptivo y del sentido lenguajario (langagier), al igual que su diferencia. Por ello, en el presente artículo buscaremos alcanzar "el

3341 Merleau-Ponty, M., Le visible et l'invisible, Paris: Gallimard, 1964, p. 227.

2 "Hemos intentado un análisis del mundo percibido que lo distingue en lo que tiene de original en oposición al mundo de la ciencia o del pensamiento objetivo. Pero este análisis estaba con todo organizado en función de conceptos clásicos como: percepción (en el sentido de posición de un objeto aislable, determinado, considerado como una forma canónica de nuestras relaciones con el mundo); consciencia (entendiendo por esto el poder centrífugo del Sinn-gebung que encuentra en las cosas lo que ella misma ha puesto); sintesis (que supone elementos a ser reunidos) (por ejemplo, problema de la unidad de las Erlebnisse), materia y forma del conocimiento" (MerleauPonty, M., Le monde sensible et le monde de l'expression. Cours au Collège de France. Notes, 1953, Ginebra: Mêtis Presses, 2011, pp. 45-46). 
objetivo de la filosofia de la carne [que] es pensar a la vez, en el mismo gesto, la continuidad y la ruptura entre el mundo silencioso y el mundo hablante"3. El pasaje del mundo del silencio al de la expresión se dará como sublimación de un orden -el perceptivo- en otro -el del lenguaje- y tal sublimación, que tiene como punto de partida la carne del mundo y como punto de llegada la carne del lenguaje, será vehiculizada por la palabra (parole). Este concepto será la piedra fundamental de la relación entre un mundo y otro, pues la palabra es quien "metamorfosea las estructuras del mundo visible y se hace mirada del espíritu"4. En este concepto tendremos que "creer", ya que detrás de él se esconde una fe expresiva, fe que nos invita a pensar que la palabra hablante (parole parlante) tiene la capacidad de decir la verdad del mundo silencioso de la percepción ${ }^{5}$.

El propósito de nuestro trabajo será entonces sostener que el mundo percibido está estructurado como un lenguaje ${ }^{6}$ y que justamente por ello es posible pensar la posibilidad del pasaje del mundo mudo al mundo hablante. Sin embargo, para que este pasaje pueda culminarse no es suficiente establecer una analogía entre sus estructuras, sino que es preciso que la carne, entendida como sensible pivote, sublime lo percibido y haga migrar el sentido hacia el tejido más liviano del lenguaje.

\section{La conciencia perceptiva como expresión}

Luego de exponer a finales de 1946 Le primat de la perception et ses conséquences philosophiques frente a la Sociedad Francesa de Filosofia y de ser fuertemente cuestionado por las tesis alli sostenidas, en sus Notas preparatorias del curso sobre el mundo sensible y el mundo de la percepción, Merleau-Ponty establece como uno de sus objetivos generales "profundizar el análisis del mundo percibido mostrando que este ya supone la función expresiva"7. A lo largo del curso, el autor caracterizará a la percepción señalando que "toda percepción

\footnotetext{
Kristensen, S., "Foi perceptive et foi expressive", en: Chiasmi International, v. V (2003), p. 266. Merleau-Ponty, M., Le visible et l'invisible, p. 200.

5 En el último punto del artículo se desentrañará el sentido de esta "creencia" en la palabra y la "fe expresiva" que ella suscita.

6 "El filósofo sabe mejor que nadie que lo vivido es vivido-hablado, que, nacido en esa profundidad, el lenguaje no es un máscara sobre el Ser, sino -si se lo sabe recuperar con todas sus raíces y su frondosidad- el más válido testigo del Ser, al que no interrumpe por una inmediación sin él perfecta; sabe que la visión misma, el pensamiento mismo, como se ha dicho están 'estructurados como un lenguaje" (ibid., p. 165)

7 Merleau-Ponty, M., Le monde sensible et le monde de l'expression. Cours au Collège de France. Notes, 1953, p. 45.
} 
es percepción de algo solo mientras es también relativa impercepción de un horizonte o de un fondo, al que implica sin tematizar" y en la que "el sentido de la cosa percibida, si se la distingue de todas las otras, no está aún aislado de la constelación en que aparece; solo se pronuncia como cierta desviación (écart) respecto del nivel de espacio, de tiempo, de movilidad y, en general, de significación en que estamos establecidos; solo se da como una deformación -aunque sistemática- de nuestro universo de experiencia"9.

Esta concepción gestáltica de la percepción es la que propiciará en el autor el abandono de la noción fenomenológica clásica de conciencia en favor de la novedosa conciencia perceptiva interpretada a la luz de la función expresiva. Él mismo señalará las principales diferencias entre ambos conceptos para justificar la necesidad de renovación de esta noción clave de toda filosofia fenomenológica.

Al comenzar las Notas de curso en torno al mundo sensible y el mundo de la expresión, Merleau-Ponty señala las principales dificultades del concepto de "conciencia constituyente". En primer lugar, el autor nos dice que "tener conciencia = hacer aparecer, delante de la oscuridad del sujeto, un ser que, por esto, es elevado al valor o a la significación"10. Concebida de esta manera, dicha conciencia solo puede tener relación con sus significaciones; su poder centrífugo de Sinngebung encuentra en las cosas lo que ella misma ha puesto. Ella es ambivalente en tanto presencia inmediata a todo ser, conciencia de sus objetos y nada la separa de ellos. No obstante, también es, al mismo tiempo, distancia infranqueable en tanto los posee de punta a punta en su mirada de sobrevuelo absoluto, y jamás puede ser "tomada" por los objetos. Esta conciencia comprende el sentido como esencia, es decir, aquello que responde a la pregunta “¿qué?” y busca definir, delimitar y significar. La conciencia constituyente es, desde un principio, posición de un enunciado, algo que está sobreentendido, y ella es ya hablante. Esta noción de conciencia es la que constituye su objeto en una total claridad. Es monádica en tanto no deja abiertos horizontes más allá de sí misma, y es única no a fuerza de individualidad sino de universalidad ${ }^{11}$.

8 Merleau-Ponty, M., Résumés de cours. Collège de France 1952-1960, París: Gallimard, 1968, p. 12 .

9 Ibid.

${ }^{10}$ Merleau-Ponty, M., Le monde sensible et le monde de l'expression. Cours au Collège de France. Notes, 1953, p. 48.

${ }^{11}$ Esta noción de conciencia que describe Merleau-Ponty es la que le atribuye al primer Husserl y al Sartre escritor de La trascendencia del ego (cf. ibid., p. 55). 
Analogía y sublimación: una respuesta al problema del pasaje del silencio...

Esta concepción estática y positiva de la conciencia será firmemente rechazada y reinterpretada a la luz de la fenomenología genética de Husserl.

En contraposición, Merleau-Ponty caracteriza a la conciencia perceptiva, a través de estas tres notas principales y distintivas en relación a la noción clásica de conciencia. En primer lugar, la conciencia perceptiva no se relaciona con valores o significaciones, sino que lo hace con seres existentes. Hay un sobrepasamiento ontológico (empiètement ontologique) de las cosas sobre ella. Está rodeada, es parte del mundo en el que ocupa un punto de vista y no se encuentra absolutamente fuera del ser que nos presenta. Las cosas toman posesión del cuerpo para hacerse percibir: "es desde el interior del mundo que yo percibo y ni contorno, ni formas geométricas tendrian sentido de otra manera. [Hay] relación de connivencia entre la cualidad y mi campo sensorial"12, relación expresiva entre los sensibles y el órgano perceptor. De este modo la proximidad y la distancia no son irreconciliables. Existe, por un lado, una proximidad entre ella y los existentes que está fundada sobre el sistema de equivalencias que se establece entre mi esquema corporal y la estructura del ser sensible, sobre la "relación expresiva" que hay entre mi manera de ser y la de la cosa. Sin embargo, esta proximidad no puede reducir la distancia que separa los campos análogos que constituyen la manera de ser de las cosas y mi manera de ser. No se posee el ser como tal sino que él resuena en mí y yo vibro al unísono con él: yo lo vivo sin jamás poseerlo ${ }^{13}$.

En segundo lugar, el sentido aquí no es esencia sino diferencia. El existente, en tanto que percibido, otorga un sentido común tácito que se revela en la armadura del paisaje en el que el sentido de una cosa percibida no está aislado de la constelación. El sentido -ya perceptivo- más que estar subsumido debajo de una esencia o significación, se da en el encuentro cuerpo-mundo como modulación de una cierta dimensión, como modo de desviación o diferencia sobre un nivel ${ }^{14}$. La conciencia perceptiva no es aquella conciencia de una figura

\footnotetext{
${ }_{12}$ Merleau-Ponty, M., Le monde sensible et le monde de l'expression. Cours au Collège de France. Notes, 1953, p. 56.

${ }^{13}$ Cf. Saint Aubert, E., "Prólogo", en: Merleau-Ponty, M., Le monde sensible et le monde de l'expression. Cours au Collège de France. Notes, 1953, p. 16.

${ }^{14}$ Respecto a la idea de considerar el sentido no como esencia sino como diferencia (écart), véase: Alloa, E., "The Diacritical Nature of Meaning: Merleau-Ponty with Saussure", en: Chiasmi International, v. XV (2014), pp. 167-179. Se buscará allí sostener que Merleau-Ponty elabora -a partir de la lectura de Saussure- "una concepción del sentido que ya no es pensada en términos de referencialidad, sino en los términos de una articulación diacrítica inmanente" (ibid., p. 168) en tanto que "el sentido emerge no de lo sustancial, sino de la relación lateral" (ibid., p. 169). Véase también: Kearney, R., "Ecrire la chair: L'expression diacritique chez Merleau-Ponty", en: Chiasmi International, v. XV (2014), pp. 183-198. Este artículo está dedicado a sostener que todas las
} 
en ausencia de cualquier fondo, sino que es conciencia de una desviación, de un movimiento, que se da como modulación sobre un nivel, como cambio que se distingue sobre un paisaje.

En tercer y último lugar, "la conciencia perceptiva no es posición de un enunciado, ella es tácita"15. El sentido de lo percibido no está hecho de significaciones libres y que existen por sí, sino que "hay equivalencias, una sintaxis del contexto, una virtud de la coexistencia o de la proximidad espacial"16. La significación está unida e implicada en lo sensible. Existe un sentido no hablante, un "mutismo" de la percepción en la que ella se perfila como una suerte de lenguaje ${ }^{17}$.

Es a partir de esta consideración de las propiedades características de la conciencia perceptiva que Merleau-Ponty afirmará que "la percepción es expresión, expresión del mundo"18, entendiendo por "expresión" la "propiedad que tiene un fenómeno, por su disposición interna, de hacer conocer otro fenómeno que no está o incluso nunca ha sido dado"19. Percibir es ya expresar, y la percepción es la constelación en la que por desviación (écart) de un nivel se expresa un sentido oculto. Una significación aparece unida a dicha constelación ${ }^{20}$. Percibir es entonces expresar, son dos caras de la misma moneda. El acto en que percibo es aquel en que se expresa un fenómeno que aún permanecía oculto o no dicho: el sentido de la cosa percibida que se desvía sobre el nivel del paisaje en que aparece.

A partir de la analogía que ya comienza a esbozarse entre ambos campos entendemos la centralidad que cobrará la comprensión de la estructuración diacrítica del sentido. Ella es la que nos permitirá establecer una continuidad entre ambos mundos y, como bien señala E. Alloa: "Merleau-Ponty le da un nombre a este andamiaje de la diacriticidad: la carne"21. Detengámonos entonces en esta noción que será la que permitirá abrir la puerta que comunica un mundo con el otro.

formas de expresión comparten la función diacrítica y que "el sentido se encuentra alli donde la expresión diacrítica cruza la experiencia de la carne” (ibid., p. 183).

15 Merleau-Ponty, M., Le monde sensible et le monde de l'expression. Cours au Collège de France. Notes, 1953, p. 50.

16 Ibid., p. 57.

17 Cf. ibid., pp. 49-58.

18 Ibid., p. 48.

19 Ibid.

20 "Lo sensible nos habla un cierto lenguaje que todos comprendemos como si entre nuestro aparato perceptivo y él estuviera establecido un pacto lingüístico” (ibid., p. 50).

${ }^{21}$ Alloa, E., "The Diacritical Nature of Meaning: Merleau-Ponty with Saussure", p. 174. 


\section{La carne sensible pivote}

A lo largo de las páginas de Lo visible y lo invisible encontramos a MerleauPonty esforzándose constantemente por desentrañar el sentido de la carne. El problema que este concepto presenta es que no parece ser susceptible de una delimitación o definición estricta, pues no puede ser encasillado dentro de las categorias clásicas de la filosofía ${ }^{22}$. Es así que, más que definir, lo que podemos hacer es señalar y delinear, conjuntamente con Merleau-Ponty, sus notas esenciales. Por ello, en este segundo apartado del artículo nos avocaremos a caracterizar sucintamente la noción de carne que será el elemento constitutivo común tanto del mundo, como del cuerpo y del lenguaje y, por ende, se perfilará como el salvoconducto de la sublimación del sentido perceptivo en el sentido lenguajario.

La carne designa para Merleau-Ponty un tipo de ser que "no tiene nombre en ninguna filosofia" 23 ya que "no es materia, no es espíritu, no es sustancia"24. La carne es "una cosa general, a medio camino entre el individuo espaciotemporal y la idea" 25 , y debe ser pensada con el antiguo término de "elemento" que utilizaban los griegos para referirse al agua, al aire, etcétera. La carne no es unión o compuesto de dos sustancias; por el contrario, es la noción última que quiere sostener la relación entre el cuerpo y el mundo, el cuerpo y el espíritu, entre lo visible y el vidente, que no pueden ser tajantemente separados sino que tienen que ser comprendidos teniendo en cuenta que "hay inserción reciproca y entrelazamiento de uno en el otro"26.

En una nota de trabajo de junio de 1960, Merleau-Ponty reflexiona en torno a cómo deben ser comprendidas las relaciones referidas más arriba. El filósofo quiere romper de una vez por todas con el pensamiento dicotómico que ha intentado siempre instalar la polaridad cuerpo-espíritu. Por ello afirma que "hay un cuerpo del espíritu y un espíritu del cuerpo y un quiasmo entre ellos"27. Este quiasmo se da en la carne (chair), noción a la que Merleau-Ponty decide

\footnotetext{
${ }^{22}$ Para profundizar en el devenir histórico de esta noción, $c f$. Carbone, M., "Flesh: Towards the History of a Misunderstanding”, en: Chiasmi International, v. IV (2002), pp. 49-62. En este artículo encontramos una breve historia que gira en torno al concepto de carne (flesh, chair). Carbone buscará presentar dicha noción recogiendo las palabras de aquellos pensadores que se interesaron por este término como Merleau-Ponty, Husserl, Franck, Nancy, Derrida y Henry. Finalmente, el autor reforzará lo que para él constituye lo esencial del término: la reversibilidad.

${ }^{23}$ Merleau-Ponty, M., Le visible et l'invisible, p. 191.

24 Ibid., p. 181.

25 Ibid., p. 182.

${ }^{26}$ Ibid., p. 180.

27 Ibid., p. 307.
} 
apelar para no permanecer en el lenguaje propio del ya mentado pensamiento tradicional: "hay que pensar la carne no a partir de las substancias, cuerpo y espíritu, pues entonces sería la unión de contradictorios, sino, decíamos, como elemento, emblema concreto de una manera de ser general" 28 . Es la carne la que nos permitirá elaborar el problema de estas dos regiones de un modo novedoso ${ }^{29}$.

La noción de carne se perfilará como bisagra o pivote entre un mundo y el otro. Es lo que propiciará el pasaje entre una dimensión y otra al ser la llave para abrir la puerta entre la percepción y el lenguaje. Entre estos dos mundos hay discontinuidad y continuidad; el quiebre se visibiliza en el pasaje de uno a otro, en el cambio que implica el pasaje del logos endiathetos al logos prophorikos. La continuidad se hace patente en la noción de sentido, noción unitaria que permanece tanto en uno como otro y que permite pensarlos debajo del mismo horizonte: "el problema de las relaciones entre percepción y lenguaje no es aquel del pasaje entre dos órdenes sin medida común, sino más bien el de la interpretación del origen común en la esencia comprendida como la textura de la experiencia, su estilo, mudo al principio, proferido después"30

Tanto percepción como lenguaje no son, a fin de cuentas, órdenes distintos que no tienen contacto entre sí, sino que ambos proceden de un origen común que se encuentra en la esencia tal como es comprendida por MerleauPonty hacia el final de su obra en Lo visible y lo invisible. Para el fenomenólogo francés la esencia "será aquello sin lo cual no habría mundo, ni lenguaje, ni cosa alguna" ${ }^{1}$. En otras palabras, la esencia es el sentido tanto de lo percibido como de lo hablado y lo pensado.

Merleau-Ponty sostiene que vivimos en complicidad con el mundo, la verdad y el ser y, cuando nos volvemos hacia ellos y nuestro pensamiento los dobla, constatamos que el sentido del pensamiento es sentido del sentido. Es decir, existe una unidad común que sostiene tanto el sentido de mi pensamiento y mi lenguaje, como también el sentido del mundo, sentido que no duplico ni copio, sino que des-pliego. La esencia es lo que sostiene aquel sentido, es aquello

\footnotetext{
${ }^{28}$ Ibid., p. 191.

29 "La noción esencial para una filosofia tal es la de la carne, que no es el cuerpo objetivo, que tampoco es el cuerpo pensado por el alma (Descartes) como suyo, que es lo sensible en el doble sentido de lo que uno siente y lo que siente. Lo que uno siente = la cosa sensible, el mundo sensible = el correlato de mi cuerpo activo, lo que le 'responde' - Lo que siente $=$ no puedo plantear un único sensible sin plantearlo como arrancado de mi carne, extraído de mi carne, y mi carne es uno de los sensibles en el cual se hace inscripción de todos los otros, sensible pivote en el cual participan todos los otros, sensible-llave, sensible dimensional" (ibid., pp. 307-308).

${ }^{30}$ Kristensen, S., "Foi perceptive et foi expressive", p. 266.

${ }^{31}$ Merleau-Ponty, M., Le visible et l'invisible, p. 143.
} 
sin lo cual no habría ni mundo, ni lenguaje, ni cosa alguna y que me permite establecer una continuidad entre estas dimensiones.

La esencia se encuentra sostenida por el tejido de la experiencia, por la carne del tiempo. Toda ideación posible, cualquier invariante que hallemos en la cosa, es sostenido por la cosa misma ${ }^{32}$. La esencia está enraizada en los hechos y el sentido perceptivo que está apresado allí puede ser sublimado en el lenguaje y en el pensamiento porque, como más arriba afirmábamos, percepción y lenguaje surgen de un origen común: la esencia ${ }^{33}$.

Esta concepción de la esencia que describimos se encuentra en aquella "región salvaje"34 en que las ideas aparecen articuladas en los ejes secretos de nuestra experiencia. Son aquellas esencias carnales, alógicas, que no pueden desprenderse del hecho en que aparecen ya que no nos pueden ser dadas independientemente de lo sensible, son esencias salvajes ${ }^{35}$.

Históricamente hemos llevado a cabo una bifurcación del hecho y la esencia porque ello es lo que imponía el pensamiento que miraba el ser de frente, pero hemos aprendido que: "Al no estar más el Ser ante mí, sino rodeándome y, en un sentido atravesándome, y al no hacerse mi visión del Ser desde otra parte sino desde el medio del Ser, los pretendidos hechos, los individuos espacio-temporales, se encuentran desde el inicio montados sobre los ejes, los pivotes, las dimensiones, la generalidad de mi cuerpo; y las ideas, por ende, ya incrustadas en sus articulaciones"36.

La esencia no se halla entonces ni encima ni debajo de las apariencias, sino que está incrustada en la carne del mundo que, a su vez, está íntimamente

\footnotetext{
32 "toda ideación es sostenida por ese árbol de mi duración y las duraciones; esa savia ignorada nutre la transparencia de la idea" (ibid., p.148).

33 "Nuestro tratamiento de los hechos permanece diferente del tratamiento inductivo o científico. No es cuestión para nosotros de considerar la palabra o el pensamiento como la simple suma de hechos de la lengua o de los hechos del pensamiento, tal como ellos están producidos aquí o ahí, en tal o tal fecha. En cada uno de ellos, nosotros ensayamos captar eso que retoma y sublima lo precedente, anticipa lo siguiente, la emergencia de una estructura, de un campo de experiencia, que producen, más que un acontecimiento, una institución" (Merleau-Ponty, M., "Titres et travaux. Projet d'enseignement", en: Parcour deux 1951-1961, Langrasse: Verdier, 2000, pp. 24-25.

${ }^{34}$ Merleau-Ponty, M., Le visible et l'invisible, p. 152.

35 "La esencia salvaje no es una idea positiva, no podemos asignarle un $\square$ trazado $\square$, es decir una regla de experiencias, una inteligencia anónima se fija fines y ordena la naturaleza según planes bien definidos. La esencia es un fenómeno de ecos entre variaciones contingentes completamente originales de suerte que la unión entre ellas permanece mágica, sin principio positivo. Se trata de un sentido diacrítico, negativo y abierto, que tomará nuevas inflexiones en el corazón de próximas variaciones" (Dufourcq, A., Merleau-Ponty: Une ontologie de l'imaginaire, Dordrecht: Springer, 2012, p. 53).

${ }^{36}$ Merleau-Ponty, M., Le visible et l'invisible, pp. 151-152.
} 
articulada con la carne de mi cuerpo. Según Mauro Carbone, la carne debe ser entendida como la condición de posibilidad de la comunicación de la experiencia ${ }^{37}$. Esta noción es central para comprender la migración del sentido de un mundo al otro y funcionará como la bisagra que hace posible la sublimación de la experiencia en el lenguaje.

La carne es el lugar propicio para que se dé el quiasmo entre ambas dimensiones, y es en ese punto inaccesible en que la carne del lenguaje se incrusta (empiète) en la carne del mundo, posibilitando sublimar la experiencia del logos endiathetos al logos prophorikos. El quiasmo es comprendido por Merleau-Ponty como un "intercambio... de lo que percibe con lo percibido: lo que comienza como cosa termina como conciencia de la cosa, lo que comienza como 'estado de conciencia' termina como cosa" 38 . Esta relación no puede ser explicada recortando la experiencia entre la región del para-sí y la del en-sí: "es necesaria una relación con el Ser que se haga desde el interior del Ser"39.

Será la carne quien establezca esta relación, y más precisamente, la carne del lenguaje: la palabra. Ella, que es el cuerpo que realiza la milagrosa promoción del Ser a la conciencia porque es uno de los visibles que se ve a sí mismo y por esto se hace luz natural y abre a lo visible su interior, "es también órgano o resonador de todos los demás y, por tanto, coextensiva a lo pensable. La palabra es parte total de las significaciones como la carne de lo visible, como ella, relación al Ser a través de un ser"40.

\section{II.1. La palabra: relación al Ser a través de un ser}

El quiasmo entre el mundo mudo y el mundo de la expresión -intercambio en que lo visible se torna invisible y viceversa- sucede en la palabra (parole) entendida como acontecimiento semiótico en que se instituye una nueva significación. Es en la palabra en que se da aquella relación al Ser a través de un ser que clamaba ser hecha desde su interior y no desde fuera. La palabra es el elemento constitutivo de la carne del lenguaje, ella forma parte de lo visible y al mismo tiempo se prolonga en el mundo inteligible. El lenguaje hablante, conformado por palabras conquistadoras que usurpan un territorio foráneo que permanecía innombrado, es la dimensión del sujeto en la que se

37 Cf. Carbone, M., "Flesh: Towards the History of a Misunderstanding", p. 50.

${ }^{38}$ Merleau-Ponty, M., Le visible et l'invisible, p. 264.

39 Ibid. La referencia de Merleau-Ponty a la ontología sartreana no hace más que reafirmar la idea que intentamos sostener, aquella que afirma que no puede seguir haciéndose un análisis del mundo percibido mirando el ser de frente, como ob-jeto.

40 Ibid., pp. 155-156. 
da la sublimación de la experiencia muda del encuentro con el mundo, y es el lugar del quiasmo en que el mundo mudo se entrecruza y se confunde con el mundo de la expresión. La palabra es un ser del mundo visible y al mismo tiempo abre a lo invisible. Tomemos una cita que, aunque extensa, intenta explicar el problema al que nos referimos: "El envolvimiento (enroulement) en el sujeto viviente de lo visible y de lo vivido sobre el lenguaje, del lenguaje sobre lo visible y lo vivido, los intercambios entre las articulaciones de su paisaje mudo y las de su palabra, en fin, ese lenguaje operante que no necesita ser traducido en significaciones y pensamientos, ese lenguaje-cosa que vale como arma, como acción, como ofensa y como seducción, porque hace aflorar todas las relaciones profundas de lo vivido donde se ha formado, y que es el de la vida y de la acción, pero también el de la literatura y la poesía, entonces ese logos es un tema absolutamente universal, es el tema de la filosofia. Ella misma es el lenguaje, descansa en el lenguaje; por eso no la descalifica para hablar del lenguaje ni para hablar del pre-lenguaje y del mundo mudo que los dobla: por el contrario, ella es lenguaje operante, ese lenguaje-alli que no puede saberse sino desde adentro, por la práctica, que está abierto a las cosas, llamado por las voces del silencio, y continua un ensayo de articulación que es el Ser de todo ser"41.

El lenguaje operante del que nos habla aquí Merleau-Ponty hace referencia al lenguaje vivo, el cual despliega un dinamismo creativo que busca constantemente instituir nuevas significaciones para decir el mundo. Este lenguaje es el que se sirve de las palabras instituidas y las utiliza como herramientas para decir más. Las palabras que entrañan un sentido que ha nacido en el entramado de las relaciones en que vive el sujeto son entendidas como ese "lenguaje-cosa" que vale como un instrumento de acción ${ }^{42}$. Estas palabras carnales son parte del mundo visible y, simultáneamente, abren al mundo inteligible. La carne del lenguaje-cosa -sus palabras carnales- entrañan el sentido que está establecido por las relaciones en que han nacido y que luego ellas harán aflorar en la situación en que el sujeto hablante o el escritor las utilice para dirigirse hacia aquello que quiere expresar ${ }^{43}$. Son palabras cargadas de un sentido instituido

\footnotetext{
41 Ibid., pp. 165-166.

42 El sentido en que debe interpretarse este lenguaje-cosa hace referencia al hecho de que las palabras tienen una acción efectiva sobre el mundo, como un arma, o un gesto, el poder de seducción, etcétera. De ninguna manera debe interpretarse esta expresión con el fin de ver en el lenguaje una cosa que existe del mismo modo que los objetos del mundo.

${ }^{43}$ Es menester remarcar el juego intencionalmente construido que se propone de aquí en adelante entre las expresiones "entrañar", "palabras carnales", "palabras preñadas": solo lo carnal puede
} 
que hacen aflorar las relaciones profundas en que han sido dadas a luz y que ahora sostienen y, son también, aquellas palabras de la literatura, significaciones que nos abren un mundo invisible ${ }^{44}$.

Para introducirnos en el problema de la sublimación del mundo mudo en el mundo de la expresión -verdadero problema de la filosofia que llamada por las voces del silencio continúa ensayando la articulación que es el Ser de todo ser- es necesario tener en cuenta que el lenguaje debe considerarse siempre en su estado naciente, creativo, y no como conjunto de significaciones cerradas e univocas. Las palabras a las que hacemos referencias no son conceptos vacíos de algún mundo inteligible, sino que son palabras carnales, palabras que -como dice Merleau-Ponty-actúan, ofenden, seducen. Estas son las palabras que pertenecen al mundo visible, que forman parte del Ser y que por ello pueden establecer una relación desde el ser. Es en ellas donde se da el quiasmo entre lo visible y lo invisible ya que, como el cuerpo que es una cosa entre las cosas pero al mismo tiempo el que las ve y toca, de ellas podemos decir que son seres entre los seres pero al mismo tiempo pueden ser su asilo. La palabra acoge el mundo silencioso y metamorfosea sus estructuras para abrir el mundo de la expresión: "Cuando la visión silenciosa cae en la palabra y cuando, a la inversa, la palabra, abriendo un campo de lo nombrable y lo decible, se inscribe alli, en su lugar, según su verdad, en resumen, cuando ella metamorfosea las estructuras del mundo visible y se hace mirada de la mente, intuitus mentis, es siempre en virtud del mismo fenómeno fundamental de reversibilidad que sostiene tanto a la percepción muda como a la palabra, y que se manifiesta tanto por una existencia casi carnal de la idea, como por una sublimación de la carne" ${ }^{\text {45 }}$.

La reversibilidad es la característica esencial de la palabra en que Merleau-Ponty se apoyará para sostener la posibilidad de la migración de un mundo a otro. La relación que existe entre el sentido de lo percibido y el sentido del lenguaje es una relación dialéctica en que silencio y lenguaje constituyen las dos dimensiones de esta verdad ${ }^{46}$.

llevar algo en las entrañas, solo lo carnal puede estar preñado de un sentido.

${ }^{44}$ A esto es a lo que se refería Merleau-Ponty en Fenomenología de la percepción al explicar que: "Descubrimos bajo la significación conceptual de las palabras una significación existencial que no solo traducen, sino que las habita y les es inseparable" (Merleau-Ponty, M., Phénoménologie de la perception, Paris: Gallimard, 1945. p. 212)

${ }_{45}$ Merleau-Ponty, M., Le visible et l'invisible, p. 200.

${ }^{46}$ Cf. Ralón de Walton, G., "La reversibilidad del silencio y el lenguaje", en: Agora, v. XI, 1 (1996), p. 161 . 


\section{Analogia y sublimación}

Este tercer apartado del artículo estará estructurado en dos partes principales (III. 1 "Analogia” y III. 2 "Sublimación”) que vienen a sintetizar lo expuesto en los apartados I y II. Las nociones de analogía y sublimación nos permitirán pensar el ya anunciado binomio continuidad-discontinuidad que existe entre el mundo de la percepción y el mundo de la expresión. La continuidad será trabajada en la primera parte, que se titula "Analogía". Aquí sentaremos las bases del problema y mostraremos que la estructuración de ambos mundos hace posible el establecimiento de una continuidad entre un orden y el otro. La posible prolongación de lo percibido en lo expresado está sustentada en la concepción unitaria del sentido entendido de modo diacrítico. Para la exposición de este problema nos remitiremos a la noción de conciencia perceptiva ya expuesta en el apartado I. La renovación de conciencia entendida como conciencia perceptiva o expresión será lo que nos habilitará, conjuntamente con la concepción diacrítica del sentido, a establecer una analogía entre ambos mundos. La discontinuidad será encarada en la segunda parte titulada "Sublimación". Allí retomaremos lo expuesto en el apartado II: la noción de carne nos permite comprender la discontinuidad, al ser la carne pivote entre ambos mundos que, aunque análogos, son distintos. La palabra se manifestará como esa región de la carne que posibilitará pensar una vía de sublimación entre estos dos mundos diversos, pero no por ello incomunicados.

\section{1. Analogia}

Existe un "juego mimético" entre la estructura del lenguaje y la estructura de la percepción: ambos mundos no son equivalentes pero sí análogos. El lenguaje recrea e imita de modo semejante el movimiento de la dinámica perceptiva $^{47}$. Cuando hablo voy tejiendo una red de significaciones que busca referirse -de modo análogo- a la red originaria y fundante de mi experiencia perceptiva. Puedo establecer entre ambos mundos una correspondencia estructural ${ }^{48}$ que

\footnotetext{
47 "Esta acción a distancia del lenguaje, que coincide con los significados sin tocarlos, esta elocuencia que los designa de manera perentoria, sin cambiarlos nunca en palabras ni hacer cesar el silencio de la conciencia, son un caso eminente de la intencionalidad corporal... A condición de que yo no reflexione expresamente sobre él, la conciencia que yo tengo de mi cuerpo es inmediatamente significativa de un determinado paisaje a mi alrededor" (Merleau-Ponty, M., Signes, Paris: Gallimard, 1960, p. 111).

${ }^{48}$ Ya en los inicios de su filosofia Merleau-Ponty esboza esta analogía entre el mundo de la percepción y el mundo de la expresión afirmando que lo expresado y la expresión tienen en común las mismas propiedades estructurales, entre ellos se da una correspondencia estructural en la que "el signo verdadero representa lo significado, no según una asociación empírica, sino porque
} 
nos invita a pensar una analogía, nos sugiere llevar a cabo un razonamiento que está basado en la semejanza de atributos que se da entre estos dos seres diferentes: el mundo percibido y el mundo expresado.

En una nota de trabajo titulada Percepción y lenguaje de octubre de 1959, Merleau-Ponty apunta: "Describo la percepción como el sistema diacrítico, relativo, opositivo, -el espacio primordial como topológico (es decir, tallado en una voluminosidad total que me rodea, donde estoy, que está detrás de mí y también ante mí...)"49. La percepción tiene una característica o nota esencial que atañe a su estructura. Esta queda definida como sistema diacrítico, relativo y opositivo, esto es, como sistema en que el sentido aparece por la diferencia o modulación que se da entre las relaciones de oposición establecidas entre los términos del sistema. El mundo percibido -como hemos señalado en el primer apartado del artículo- se encuentra estructurado como un lenguaje, es decir, como un juego de oposiciones y de relaciones entre los términos en que aparece el sentido por desviación (écart) sobre un nivel implícito. El sentido de lo percibido, la percepción de una cosa, se da en el encuentro de la carne del mundo con la carne del cuerpo. Por ello, Merleau-Ponty señala que cuando percibo no percibo un color aislado, un trozo de ser absolutamente macizo, indivisible, algo que se ofrece desnudo a la visión, sino que, al contrario, lo que se da es "algo que viene a tocar suavemente y hace resonar a distancia diversas regiones del mundo colorido o visible, cierta diferenciación, modulación efimera de este mundo, menos cosa o color, pues, que diferenciación entre cosas y colores, cristalización momentánea del ser colorido o visibilidad" 50 .

Por otro lado, podemos señalar en el mundo de la expresión una estructura análoga a la del mundo de la percepción como la que se da en el caso del lenguaje. Es interesante advertir la intención de Merleau-Ponty por establecer una notoria semejanza con el mundo de la percepción, pues tal es esta relación que el filósofo selecciona cuidadosamente las mismas palabras para caracterizar ambas estructuras. La siguiente cita, aunque algo extensa, nos permitirá vincular directamente un mundo con otro: "Definimos con Saussure los signos, no como los representantes de ciertas significaciones, sino como medios de diferenciación del encadenamiento verbal y de la palabra, como 'entidades opositivas, relativas y negativas'. Una lengua no es tanto una suma de signos,

su relación con los otros signos es la misma que la relación del objeto significado por él respecto a los otros objetos" (Merleau-Ponty, M., La Structure du comportement, Paris: PUF, 1942, p. 132).

${ }^{49}$ Merleau-Ponty, M., Le visible et l'invisible, p. 263.

50 Ibid., p. 173. 
(palabras y formas gramaticales y sintácticas) cuanto un medio metódico de discriminar unos signos de otros, y de construir de ese modo un universo de lenguaje, del que luego podamos decir -cuando llegue a ser tan preciso que pueda cristalizar una intención significativa y hacerla renacer en otro-, que expresa un universo de pensamiento"51.

Este pasaje no hace más que confirmar la analogia que queremos establecer entre ambos mundos, y en él resuena el eco de la definición de la percepción como sistema diacrítico. El lenguaje conformado por entidades opositivas, relativas y negativas, funciona como una estructura en la que el sentido aparece por la posibilidad de discriminar unos signos de otros. La emergencia del sentido que se da en la estructura del lenguaje -cuando logra precisarse- puede finalmente cristalizar una intención significativa y hacer renacer en el otro el universo de pensamiento del sujeto hablante. Este es el "juego mimético" al que nos referíamos al comienzo de este apartado. El ser del lenguaje se asemeja al ser de la percepción en tanto que sus estructuras son análogas y es por esto que podemos afirmar sin reparos que: "El lenguaje no dice nunca nada, lo que hace es inventar una gama de gestos que presentan entre sí diferencias lo suficientemente claras como para que el comportamiento del lenguaje a medida que se repite, se implica y se confirma a sí mismo, nos proporcione de manera irrecusable la traza y los contornos de un universo de sentido"52.

Tanto el lenguaje como la percepción presentan una dinámica de funcionamiento en la que la emergencia del sentido se da como modulación, como diferencia o desviación sobre un nivel. El pasaje de un sistema a otro es posible porque ambos atrapan el sentido estructurándolo del mismo modo. La continuidad que puede ser establecida reside en la concepción diacritica del sentido, principio unitario que permite pensar percepción y lenguaje debajo del mismo horizonte. La incipiente analogía entre ambos mundos que acabamos de insinuar cobra entonces su mayor fuerza a la luz de la consideración del problema de la emergencia diacrítica del sentido. Explicitar lo diacrítico en ambos mundos nos permitirá asentar definitivamente la continuidad que puede observarse entre las dos regiones.

En los ya citados cursos en torno al mundo sensible y el mundo de la expresión, Merleau-Ponty instaura dicha analogía diciendo: "Percibir una fisonomía, una expresión, es siempre usar signos diacríticos, al igual que realizar

51 Merleau-Ponty, M., La prose du monde, Paris: Gallimard, 1969, p. 45.

52 Ibid., pp. 46-47. Las cursivas pertenecen al texto original. 
con el cuerpo una gesticulación expresiva. Aquí cada signo no tiene otro valor que el de diferenciarlo de los otros"53. Tanto percibir como expresarse implican la necesidad de desentrañar el sentido diacrítico establecido por los signos. El hecho de que el signo sea diacrítico supone "que solo opera por diferencia, por un cierta separación entre él y los otros signos, y no primero evocando una significación positiva" ${ }^{4}$. Lo diacrítico debe ser entendido etimológicamente como aquello "a través de lo cual" (dia) una "separación" (diakrisis) habrá de hacerse ${ }^{55}$. Percibir las cosas y sus aspectos particulares supone advertir aquella desviación sobre el nivel que aparece en el entramado de los términos que configuran el todo de lo percibido. Expresarse es usar esos signos y disponerlos de tal manera que puedan dirigir la expresión hacia lo expresado, hacia lo que se quiere decir, modular los gestos para referirse a algo. Percepción y lenguaje, más que sistemas de referencia directa, son configuraciones que insinúan el sentido de lo que aparece o se muestra a través de ellos. Son conjuntos de signos en que se destaca un sentido que solo puede ser comprendido en la relación establecida entre estos y que nos reenvía hacia lo que se quiere mostrar o decir: "Como los signos en el lenguaje los puntos atravesados en el movimiento no tienen más que un valor diacrítico, ellos no funcionan cada uno por su cuenta anunciando un lugar sino solamente marcando un pasaje como las palabras de la frase son la huella de una intención que no hace más que transparentarse el lenguaje es apertura de un campo reorganizado, con otros contornos, otras coordenadas que aquellas del campo perceptivo natural"56.

La percepción y lenguaje operan del mismo modo, los signos diacríticos que en ellos aparecen nos envían a lo que se muestra, nos insinúan lo que se expresa: "La percepción, como el lenguaje, no es enfrentamiento de un ob-jeto. $\mathrm{El} \mathrm{ob-jeto} \mathrm{solo} \mathrm{me} \mathrm{habla} \mathrm{lateralmente,} \mathrm{i.e.} \mathrm{él} \mathrm{me} \mathrm{afecta,} \mathrm{no} \mathrm{de} \mathrm{frente,} \mathrm{sino} \mathrm{de}$ costado, despertando en mi complicidad" 57 . El lenguaje como sistema diacrítico es la continuidad de la diferenciación diacrítica de lo sensible y, abre un campo análogo al de la percepción en que este mundo es reorganizado y retomado, pero esta migración del sentido se da sin corromper el juego de equivalencias y oposiciones preexistente en la percepción: "Lo percibido y lo dicho se destacan

\footnotetext{
53 Merleau-Ponty, M., Le monde sensible et le monde de l'expression. Cours au Collège de France. Notes, 1953, p. 203.

${ }^{54}$ Merleau-Ponty, M., Signes, p. 146.

55 Cf., Alloa, E., "The Diacritical Nature of Meaning: Merleau-Ponty with Saussure", p. 171.

56 Merleau-Ponty, M., Le monde sensible et le monde de l'expression. Cours au Collège de France. Notes, 1953, p. 205. El subrayado pertenece al texto original.

57 Ibid.
} 
a partir de un campo, de un trasfondo, y de la misma manera que la forma perceptiva no es como tal separable del trasfondo ya que solo existe en la diferenciación del campo perceptivo, la diferenciación lingüística no es separable del sistema de los signos puesto que solo existe en la diferenciación del campo lingüístico"58.

Sin embargo, afirmar que en ambos campos el sentido se manifiesta diacríticamente no explica la migración de uno a otro. ¿Cómo es posible entonces expresar nuestra experiencia? ¿Cuál es el modo en que el lenguaje alcanza a decir aquello que hemos percibido? ¿Cómo es que estos dos mundos análogos que estructuran el sentido de tal modo que posibilitan la continuidad, logran finalmente hacer migrar el sentido perceptivo hacia el lenguaje?

\section{III.2. Sublimación}

La institución de una significación acontece en un determinado contexto en que la cosa es percibida, situación en que el sentido de lo percibido aparece por desviación sobre un nivel. Aquel contexto que ha visto nacer la significación es del que está preñado la palabra instituida. Este signo -instituido en la dinámica de un lenguaje operante- pasa del lado de las significaciones adquiridas para hacerse una herramienta más del cuerpo con la que decir su experiencia $^{59}$. Las palabras son paridas en medio del mundo y las conductas comunes de los sujetos hablantes, la significación naciente queda inseparablemente unida a ese mundo común en que se delinea el sentido de aquello que quiere ser expresado, el lenguaje recrea el paisaje de la percepción ya que sus elementos son el fondo sobre el que se destaca el sentido de una palabra, así como el contexto perceptivo es el nivel sobre el que se desvía el sentido de la cosa percibida. Merleau-Ponty propone entonces que: "Tratamos solamente de deshacer el tejido intencional que liga uno a otro, de volver a encontrar los caminos de la sublimación que conserva y transforma el mundo percibido en el mundo hablado (monde parlé), y este solo es posible si describimos la operación de la palabra (parole) como una tematización, como una reconquista de la tesis del mundo, análoga en su orden a la percepción y diferente de ella"60.

Todo esta dinámica instituyente de nuevas significaciones del lenguaje en la que las palabras aparecen tematizando el mundo, y entrañando las rela-

\footnotetext{
58 Ralón de Walton, G., "La reversibilidad de silencio y lenguaje según Merleau-Ponty", p. 156.

59 "La palabra, la que yo profiero o la que oigo, está preñada de un significado que es legible en la textura misma del gesto lingüístico" (Merleau-Ponty, M., Signes, p. 111).

60 Merleau-Ponty, M., La prose du monde, p. 173.
} 
ciones en las que han nacido, está orientada por lo que Merleau-Ponty llama la "significación lenguajaria (langagière) del lenguaje que realiza la mediación entre mi intención aún muda y las palabras" fundida con las palabras y las anima. Mi intención de decir lo que percibo se ve asistida por estos elementos del lenguaje que me permiten dar las pinceladas necesarias para significar el paisaje de mi experiencia.

Si utilizamos la división impuesta al lenguaje por Saussure para facilitar nuestro análisis, podemos decir que la región del lenguaje que nos permitirá comprender la sublimación es la del habla (parole). Esto es lo que Merleau-Ponty afirmaba más arriba al decir que solo es posible encontrar los caminos de la sublimación del mundo percibido en el hablado si describimos la operación de la palabra como una reconquista de la tesis del mundo. Es en el habla en el que el sujeto hace uso del referido lenguaje-cosa, aquel lenguaje que hace aflorar las relaciones en las que ha nacido y que entraña. Pero ¿cómo puede una palabra sostener las relaciones existentes en el mundo percibido? ¿Cómo podemos expresar el logos mudo en el que nos encontramos? ¿Cómo podemos comunicar al otro el sentido de lo que vivimos y pensamos?

En Lo visible y lo invisible encontramos una nota sin título de enero de 1959, en la que Merleau-Ponty afirma que el "mundo perceptivo es en el fondo el Ser en el sentido de Heidegger... y que captado por la filosofia en su universalidad, aparece como conteniendo todo lo que será dicho siempre y,

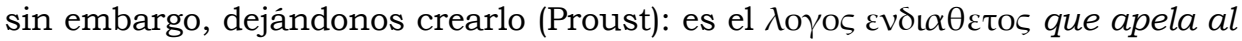

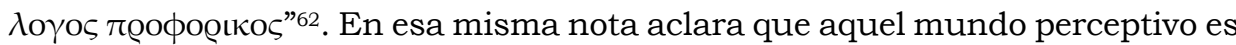
el mundo aún no tematizado que está allí como mundo de la vida (Lebenswelt) y que la filosofia no aparece en rivalidad o como antinomia de este, sino como quien lo devela ${ }^{63}$. Esta nota delimita el proyecto general en que buscaba inscribirse la filosofia merleaupontiana: llevar a cabo una recuperación del mundo de la vida a través de la tematización, de la develación que opera la filosofía al sublimar el mundo perceptivo en el mundo del lenguaje por medio de la palabra.

Es ineludible entonces la centralidad que cobra el término freudiano de sublimación, el cual se perfila como la noción que le permitirá al fenomenólogo francés pensar la relación entre percepción y lenguaje sin la intervención de la modalidad donadora de sentido del espíritu. Es menester, ya que Merleau-Ponty se apropiará del concepto de "sublimación" para pensar la migración del sentido

${ }^{61}$ Merleau-Ponty, M., Signes, p. 111.

${ }^{62}$ Merleau-Ponty, M., Le visible et l'invisible, pp. 221-222.

${ }^{63}$ Cf., ibid. 
Analogía y sublimación: una respuesta al problema del pasaje del silencio...

de un mundo a otro, detenernos brevemente en este término psicoanalítico para esclarecer, sucintamente, su génesis y definición.

\section{III.2.I. Origen y definición del término "sublimación"}

En el escrito Tres ensayos de teoría sexual ${ }^{64}$, Freud introduce, por primera vez en un texto publicado, el término "sublimación". No es casual que esta primera referencia, a la que Merleau-Ponty ha prestado especial atención, se encuentre debajo del subtítulo "Tocar y mirar"65 en el curso del desarrollo que el autor viene realizando en torno a las "Fijaciones de metas sexuales provisionales". Freud explica que las condiciones internas o externas, que pueden dificultar o posponer el alcance de la meta sexual normal, refuerzan la inclinación a demorarse en actos preliminares a partir de los cuales son constituidas nuevas metas sexuales que pueden remplazar a las normales. El análisis del tocar y el mirar, como metas sexuales provisionales, es utilizado como ejemplo para demostrar que aquellos nuevos propósitos extraños ya están esbozados en las prácticas normales. Tanto un cierto grado de uso del tacto como de la visión forman parte indispensable para el logro de la meta sexual y, siempre que el acto sexual siga adelante, estas prácticas no son consideradas perversiones. Freud afirma alli que el mirar deriva del tocar continuando el camino por el que ha de desarrollarse el objeto sexual en el sentido de la belleza y que "la ocultación del cuerpo, que progresa junto con la cultura humana, mantiene despierta la curiosidad sexual, que aspira a completar el objeto sexual mediante el desnudamiento de las partes ocultas. Empero, puede ser desviada ('sublimada') en el ámbito del arte, si uno puede apartar su interés de los genitales para dirigirlo a la forma del cuerpo como un todo"66.

En esta primera alusión, sublimar implica desviar el interés de los genitales y dirigirlo a la forma del cuerpo como un todo. La sublimación sería principalmente un proceso mediante el cual las pulsiones sexuales se desvian de sus metas y se orientan hacia otras nuevas.

\footnotetext{
64 Freud, S., "Tres ensayos de teoría sexual y otras obras", en: Obras Completas, Buenos Aires: Amorrortu Editores, v. VII, 1983, pp. 109-211.

65 S. Kristensen nos da la razón por la cual ese subtítulo es de especial importancia para la comprensión del rol que jugará la noción de sublimación en la última filosofia de Merleau-Ponty: "Encontramos en ese texto una prefiguración de la reversibilidad entre el tocar y el ver, tema central en la última parte de Lo visible y lo invisible dónde, en efecto, la visión sublima el tocar. Lo que para mi propósito es significativo es que la reversibilidad tiene una doble función: ella permite, por un lado, postular la unión entre dos sistemas sensoriales en vista de la satisfacción del deseo sexual y, por otro, ella se ubica como principio explicativo de las idealidades culturales" (Kristensen, S., "Foi perceptive et foi expressive", p. 271).

66 Freud, S., "Tres ensayos de teoria sexual y otras obras", p. 142.
} 
Más adelante, cuando Freud se ocupa del periodo de latencia sexual de la infancia y de sus rupturas, vuelve a recurrir a la utilización de la noción de sublimación. Debajo del subtítulo "Formación reactiva y sublimación", el autor nos explica que existen en el niño mociones sexuales infantiles que permanecen activas incluso en el período de latencia, pero que su energía "es desviada del uso sexual y aplicada a otros fines"67. A continuación atribuye a los historiadores la suposición de que "mediante la desviación de las fuerzas pulsionales sexuales de sus metas, y su orientación hacia metas nuevas (un proceso que merece el nombre de sublimación), se adquieren poderosos componentes para todos los logros culturales"68.

Es claro que la intención de Freud es utilizar la noción de sublimación para describir el proceso en el que se da una desviación de las pulsiones sexuales hacia una meta nueva y distinta de la que se había sostenido hasta ese momento. Además, agrega que este proceso implica la adquisición de poderosos componentes culturales. Esta característica es sumamente importante para nosotros ya que Freud ubica a la sublimación en el límite entre lo somático y lo psíquico, es decir, lo que pertenece a la pulsión sexual es desviado hacia una nueva meta en la que puede formarse un componente cultural. El concepto de sublimación, tal como Merleau-Ponty lo toma de Freud, supondrá la posibilidad de ligar lo corporal con lo cultural, la posibilidad de poner en un mismo nivel lo sensible y lo ideal. La sublimación -término más utilizado que explicado por Freud- sería aquel proceso por el cual a partir de la dialéctica entre el deseo y lo otro del deseo se formarian unidades culturales que tienen como destino ser adquisiciones intersubjetivas.

La importancia que tiene esta migración del concepto de sublimación del psicoanálisis freudiano a la fenomenología merleaupontiana, reside en el hecho de que esta puede ser considerada como el proceso por el cual los sujetos se relacionan a un mundo común y le donan sentido a su vida. La sublimación se posiciona así como la función simbólica del sujeto ${ }^{69}$.

III.2.II. La sublimación: fundamento de la reversibilidad entre percepción y lenguaje

Luego de haber aclarado sucintamente el origen y la definición del término de sublimación, podemos posicionarlo en el centro de la explicación que

\footnotetext{
${ }^{67}$ Ibid., p. 161.

${ }^{68}$ Ibid. Las cursivas pertenecen al texto original.

${ }^{69}$ Cf. Kristensen, S., Parole et subjectivité. Merleau-Ponty et la phénoménologie de l'expression, Hildesheim: Georg Olms Verlag, 2010, p. 160.
} 
Analogía y sublimación: una respuesta al problema del pasaje del silencio...

Merleau-Ponty llevará a cabo para dar cuenta del paso del mundo sensible al mundo de la expresión. La sublimación será aquello que explicitará y fundará la reversibilidad ${ }^{70}$ entre ambas dimensiones. Lejos de retomar esta noción en su vertiente sexual, el filósofo se apropiará de ella con otra intención: "por 'sublimación', Merleau-Ponty designa siempre el proceso por el cual un sentido se estabiliza y se libera del contexto que lo vio nacer para prestarse a una reproducción"71. Esto es lo esencial del concepto: la posibilidad de vehiculizar un sentido hacia otra dimensión en la que será re-producido. La sublimación, como hemos dicho anteriormente, será aquel proceso por el cual el sentido perceptivo es conservado y transformado para migrar hacia otro orden ${ }^{72}$.

Se trata entonces, como establecimos al comienzo de este apartado, de deshacer el tejido intencional que liga el mundo percibido al mundo hablado y encontrar los caminos de sublimación que conservan y transforman uno en el otro. Este paso de una dimensión a otra debe ser interpretado como una reorganización de aquello que se encuentra en una estructura dada o como realización temática del logos del mundo sensible en una arquitectónica diferente.

Nuestra argumentación tiene como fundamento una noción de sentido unitaria que reside en el concepto de carne. Es posible reconstruir en la filosofia merleaupontiana del último período una teoría carnal de las ideas. La carne es donde residen las esencias que no pueden ser separadas de los hechos en que se manifiestan. La carne del mundo se incrusta (empiète) sobre la carne del cuerpo $y$ es en ese quiasmo en el que el sentido de lo percibido puede reorganizarse para migrar al sentido del lenguaje. La articulación del silencio y la palabra deben ser pensadas teniendo en cuenta que los nudos de sentido-las ideas- no son positividades opuestas al mundo sensible, sino que, al contrario, son un nivel que no es extraño a la carne sino que le da su profundidad y dimensiones.

\footnotetext{
${ }^{70}$ La reversibilidad será el entrelazo entre las cosas y la palabra que viene a decir un modo de identidad en la diferencia, la idealidad invisible del lenguaje ilumina el mundo y pone de manifiesto su sentido, a su vez, el lenguaje surge de la dehiscencia y repliegue sobre sí misma de la carne del mundo. El lenguaje es el medio en que el mundo se refleja y se piensa. No duplica ni copia el mundo, es el modo como el sentido del mundo se despliega. Esta relación de reciprocidad en que ninguno de los elementos es inteligible por sí mismo es la relación de reversibilidad (Cf. Aspiunza, J., "El lenguaje en el Pensamiento de Merleau-Ponty" en: Eyzaguirre, S. (ed.) Fenomenología y Hermenéutica. Actas del I Congreso Internacional de Fenomenología y Hermenéutica, Santiago de Chile: RIL Editores, 2008, p. 114).

${ }^{71}$ Kristensen, S., Parole et subjectivité. Merleau-Ponty et la phénoménologie de l'expression, p. 164.

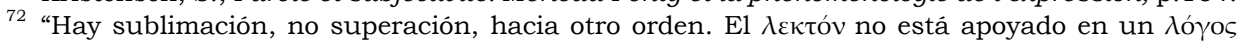
independiente del 'mundo estético"' (Merleau-Ponty, M., L'institution dans l'histoire personnelle et publique. Le problème de la passivité, le sommeil, l'inconscient, la mémoire. Notes de Cours au Collège de France 1954-1955. París: Berlin, 2003, p. 90).
} 
Merleau-Ponty explica que "como la nervadura sostiene la hoja desde dentro, desde el fondo de su carne, las ideas son la textura de la experiencia; su estilo, mudo al comienzo, proferido después"73.

El concepto que permitirá sublimar un sentido en el otro es la palabra (parole) que, como nos indicaba Merleau-Ponty, es en donde se encuentra la clave para comprender este acontecimiento. La palabra es la operación que supone una reconquista de la tesis del mundo porque ella, como carne del mundo, puede prolongar en lo invisible y abrir el mundo de la idea. El cuerpo y la palabra constituyen los modos originarios de apertura al mundo y así "como la estructura sensible no puede comprenderse sino por su relación con el cuerpo, con la carne -la estructura invisible no puede comprenderse sino por su relación con el logos, con la palabra"74. La estructura de lo sensible se sostiene en relación a la carne del cuerpo y, análogamente, la estructura de lo invisible se encuentra en tensión con la palabra.

Ambas estructuras -la del mundo sensible y la del mundo de la expresión- presentan una organización análoga y es por esto que se puede instaurar la posibilidad de pasar de una a otra. Pero para que se produzca la migración del sentido no es suficiente el establecimiento de cierto paralelismo o analogía de estructuras. El paso del mundo del silencio a la palabra se sostiene por el fenómeno fundamental de la reversibilidad que puedo establecer entre uno y otro. Esta se manifiesta "tanto por una existencia carnal de la idea como por una sublimación de la carne"75.

La sublimación del silencio en la palabra, del mundo percibido en el mundo hablante, se erige como el principio explicativo de las unidades culturales. El silencio del mundo, que es logos de la percepción reversible en palabra, encuentra su lugar en la estructura del logos proferido gracias a la sublimación que implica un entrecruzamiento, una metamorfosis de un sentido en el otro: "Se trata, por ambos lados, de la misma trasmutación, de la misma migración de un sentido esparcido en la experiencia, que abandona la carne en la que no llega a adquirir cohesión, que moviliza en provecho propio instrumentos ya investidos, y los emplea de tal manera que acaban por convertirse para él en el mismo cuerpo que necesitaba mientras pasa a la dignidad de significación expresada"76.

\footnotetext{
${ }^{73}$ Merleau-Ponty, M., Le visible et l'invisible, p. 157.

74 Ibid., p. 273.

75 Ibid., p. 200.

76 Merleau-Ponty, M., La prose du monde, p. 67.
} 
Analogía y sublimación: una respuesta al problema del pasaje del silencio...

El sentido esparcido por el mundo abandona la carne y migra hacia la carne del lenguaje, que por su disposición propia puede captarlo en sus tejidos cada vez que la palabra emerge de lo más profundo de la experiencia cristalizando la intención significativa del sujeto hablante e instituyendo una nueva significación. De este modo, sublima el sentido percibido y lo transforma en una adquisición intersubjetiva. El sentido perceptivo sublimado se convierte en una institución originaria del mundo hablado: esto sucede porque la sublimación no es otra cosa que el movimiento de pasaje de la intencionalidad corporal a la intencionalidad de la palabra ${ }^{77}$.

El pasaje del logos mudo al logos proferido es un movimiento reversible que supone que el mundo visible se conserva en la palabra y que la palabra emerge y re-produce aquel mundo. Esa reversibilidad es el principio de explicación del pasaje y también lo que caracteriza ambas instancias, a la palabra y a la percepción. Lo percibido en tanto que es parte de la carne es aquello que es decible; inversamente, lo dicho, en tanto que enraizado en la carne es, esencialmente, lo dicho de lo percibido. La sublimación será para Merleau-Ponty la posibilidad de principio de esa reversibilidad comprendida como simbolización de la carne ${ }^{78}$.

\section{III.2.III. La fe expresiva}

"Nosotros vemos las cosas mismas, el mundo es eso que vemos"79: esta es la frase con que inicia Merleau-Ponty Lo visible y lo invisible y de la que él mismo explica que "fórmulas de este tipo expresan una fe que es común al hombre natural y al filósofo desde que abre los ojos, remiten a un fundamento profundo de 'opiniones' mudas implicadas en nuestra vida" ${ }^{80}$. El objetivo de tal comienzo es el de elaborar una armoniosa reflexión filosófica en torno al problema de la percepción que uno puede hallar al recorrer las primeras páginas del libro. Podemos decir que este tema ha ocupado al fenomenólogo francés a lo largo de toda su obra. Sin embargo, allí es reelaborado a partir de una noción que nos importa especialmente: la fe $(f \circ)^{81}$.

77 Kristensen, S., Parole et subjectivité. Merleau-Ponty et la phénoménologie de l'expression, p. 166.

${ }^{78}$ Cf. ibid., p. 170.

79 Merleau-Ponty, M., Le visible et l'invisible. p. 17.

80 Ibid.

81 En Fenomenología de la percepción, Merleau-Ponty ya se había referido a la noción de fe en diversas ocasiones, sobre todo ligada a la percepción (por ejemplo en Merleau-Ponty, M., Phénoménologie de la perception, pp. 371-372 y p. 468). Sin embargo, esta noción, aunque es utilizada en el mismo sentido, no tiene alli el rol central que si puede advertirse en Lo visible y lo invisible donde desarrollará mucho más extensamente. 
El primer capítulo titulado "Reflexión e interrogación" inicia con un subtítulo que reza "La fe perceptiva y su oscuridad". En estas páginas el filósofo busca poner de manifiesto el fenómeno de la percepción y su misteriosa naturaleza. Remitiéndose a San Agustín, quien afirmaba que el tiempo era familiar para cada uno pero que nadie podía explicarlo a los demás, nos dice que la misma característica debe ser aplicada al mundo. Vivimos en íntima relación con este y nadie dudaría de que vemos las cosas mismas y el mundo es aquello que vemos. Sin embargo, cuando nos preguntamos qué es ver, qué es cosa o mundo, entramos en un laberinto de dificultades y contradicciones.

Históricamente el filósofo ha querido recorrer este laberinto y para ello ha elaborado constantemente nuevos conceptos que le permitan designar aquella evidencia del mundo que se da en el contacto cotidiano. El problema que una y otra vez se presenta y representa de diversos modos es aquel que, en el afán de explicar esa intimidad en que vivimos con el mundo -la evidencia indubitable del sujeto que está en el mundo-, siempre hace devenir la reflexión en pensamientos aparentemente más sofisticados en los que el hombre natural ya no se reconoce.

La verdadera tarea del filósofo no sería entonces aquella de intercambiar el mundo de la experiencia por las significaciones que lo reflejan: la filosofia no puede ser considerada un léxico a la que debemos imponerle la tarea de buscar los sustitutos verbales del mundo. La ocupación del filósofo es la de interrogarse, no en pos de negar el mundo intercambiando el ser por el "puede ser", sino para desplegar una interrogación en la que se haga lugar a nuestra visión del mundo y a las paradojas que ella supone. En palabras de MerleauPonty, su tarea parte de la premisa de que "son las cosas en sí mismas, desde el fondo de su silencio, lo que la filosofia quiere conducir a la expresión"82.

Este itinerario de pensamiento lleva a Merleau-Ponty a dar mayor importancia a la noción de fe, una fe que como él anota al margen del manuscrito "no es la fe en el sentido de decisión sino en el de lo que es antes de toda posición"83. Este elemento al que nuestro autor recurre no busca desentrañar el misterio de aquella evidencia ingenua del mundo, no quiere recorrer el laberinto. Por el contrario, intenta referir, sin oscurecer, aquel milagro que es la evidencia

\footnotetext{
${ }^{82}$ Merleau-Ponty, M., Le visible et l'invisible, p. 18.

83 Ibid., p. 17.
} 
Analogía y sublimación: una respuesta al problema del pasaje del silencio...

ingenua de saber que nosotros vemos las cosas mismas y el mundo es aquello que vemos ${ }^{84}$.

Nos hemos remitido a la noción de fe perceptiva porque es la herramienta a la que Merleau-Ponty recurre a la hora de responder a las antinomias que supone la elaboración de una filosofia reflexiva del ser bruto, que descubre las insuficiencias y, al mismo tiempo, la necesidad del proceso reflexivo. Esta fe nos ayudará a pensar también las antinomias de una fenomenología de la expresión que busca que "la experiencia pura y por así decirlo aún muda, sea llevada a la expresión pura de su propio sentido". La contradicción en una filosofia tal implica, por un lado, el hecho irrefutable de que la palabra es derivada de la percepción y, por el otro, la transformación (bouleversement) que ella introduce al expresarla. Es, en otras palabras, la paradoja del lenguaje que no es primero siquiera autónomo y, sin embargo, es siempre en él en donde se dice aquello que lo precede, su fundamento. ¿Es la palabra dicha sobre el mundo palabra verdadera? ¿Lo dicho sobre la percepción es lo percibido? Esta es una cuestión insalvable, es la aporía a la que nos arroja toda reflexión que busque elucidar el fenómeno de la expresión: un círculo vicioso en el que sabemos que la percepción suscita la expresión. Sin embargo, expresar -único modo de manifestar lo percibido- parece transformar lo vivido.

No casualmente comenzamos este artículo explicando que el pasaje de un mundo a otro iba a darse como sublimación y que la vehiculización de la migración del sentido iba a ser llevada a cabo por la palabra (parole), concepto en el que tendríamos que "creer". Llegando al final es preciso entonces que esa creencia sea "explicada". La "fe expresiva" es un concepto que elabora S. Kristensen para responder a las antinomias de una filosofia del sentido, filosofia que supone que la unión de la palabra a la realidad -el hecho de que ella esté destinada a decir la verdad del mundo- es un hecho tan incontestable como imposible de probar85.

La palabra hablante es palabra conquistadora, y por su carácter indeterminado puede significar con exactitud. Ella introduce un acontecimiento inédito sin el cual no podríamos tener acceso a aquello sobre lo que se funda.

\footnotetext{
84 “Para nosotros, la 'fe perceptiva' envuelve todo lo que se ofrece al hombre natural en original en una experiencia-fuente, con el vigor de lo que es inaugural y presente en persona, según una visión que, para él, es definitiva y no podría concebirse más perfecta o más próxima, ya se trate de las cosas percibidas en el sentido corriente de la palabra o de su iniciación al pasado, al imaginario, al lenguaje, a la verdad predicativa de la ciencia, a las obras de arte, a los otros, o a la historia" (ibid., pp. 207-208).

$85 C f$. Kristensen, S., "Foi perceptive et foi expressive", p. 276.
} 
La fe expresiva, si asumimos la posición de Kristensen, envolvería al sujeto hablante de tal modo que le haría concebir como posición primera, anterior a todo análisis, el hecho de que las palabras nombran mi relación con el mundo ${ }^{86}$.

Al hablar digo aquello que percibo y puedo comunicar mi experiencia hasta lograr que la intención significativa que surge del encuentro de mi carne con la carne del mundo logre cristalizarse de tal modo que pueda ser reasumida por otro. Así como la fe perceptiva es aquello que se da antes de toda posición, es decir, la certeza incontestable de que vemos la cosas mismas y el mundo es aquello que vemos (certeza que luego será necesariamente oscurecida por la reflexión filosófica), la fe expresiva es la certidumbre, anterior a toda reflexión, de que las palabras están ligadas de tal modo a la experiencia que al hablar lo que nombro es el mundo de mis vivencias. Tanto la fe perceptiva como la expresiva suponen que estamos en el mundo y en el lenguaje, respectivamente, antes de tematizarlo, antes de volver sobre este.

Todo intento de conceptualización de esta situación inicial del sujeto en que se encuentra en una silenciosa coincidencia con el mundo, es ilícito. Desde el momento en que el lenguaje conceptual quiere envolver dicho silencio inicial, él lo desgarra. Será entonces un lenguaje diferente el que posibilitará el salvoconducto a la percepción silenciosa de la que nace la expresión. Esto explicaba Merleau-Ponty en una nota de febrero de 1959 titulada "Genealogía de la lógica, Historia del ser, Historia del sentido" al escribir: "Tomar posesión del mundo del silencio, tal como lo efectúa la descripción del cuerpo humano, ya no es ese mundo del silencio, es el mundo articulado, elevado al Wesen,

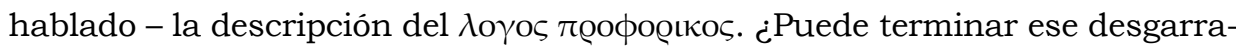
miento de la reflexión (que sale de sí mismo queriendo entrar en sí mismo)? Haría falta un silencio que envuelva a la palabra nuevamente después de que uno se haya percatado de que la palabra envolvía al pretendido silencio de la coincidencia psicológica. ¿Qué será ese silencio? Así como la reducción, finalmente, no es para Husserl inmanencia trascendental, sino develamiento de la Weltthesis, ese silencio no será lo contrario al lenguaje"87.

Ese silencio que no será contrario al lenguaje y que nos permitirá llevar la experiencia a la expresión de su propio sentido, es el silencio al que nos

\footnotetext{
86 "Hablamos y comprendemos la palabra mucho antes de aprender por Descartes (o de descubrir por nosotros mismos) que nuestra realidad es el pensamiento. Al lenguaje, en el que nos instalamos, aprendemos a manejarlo de una supuesta forma antes de aprender por la lingüística los principios inteligibles (suponiendo que ella los enseñe) en los cuales 'se apoyan' nuestra lengua y cualquier lengua" (Merleau-Ponty, M., Le visible et l'invisible, p. 28).

${ }^{87}$ Merleau-Ponty, M., Le visible et l'invisible, p. 230.
} 
conducen, de modo indirecto y lateral, los signos del lenguaje literario. Las palabras conquistadoras instituidas por la literatura son aquellas que dicen susurrando y nos enseñan que "hablar poéticamente del mundo es casi callarse"88.

Recibido: $12 / 04 / 2016$

Aceptado: 30/06/2016

\section{Bibliografia}

Alloa, E., "The Diacritical Nature of Meaning: Merleau-Ponty with Saussure”, en: Chiasmi International, v. V, 2014.

Aspiunza, J., "El lenguaje en el pensamiento de Merleau-Ponty", en: Fenomenología y Hermeneútica. Actas del I Congreso Internacional de Fenomenología y Hermenéutica, Eyzaguirre Tafra, S. (ed.), Santiago de Chile: RIL Editores, Universidad Andrés Bello, 2008.

Carbone, M., "FLESH: Towards the History of a Misunderstanding", en: Chiasmi International, v. IV, 2002. https://doi.org/10.5840/chiasmi2002413

Dufourcq, A., "Merleau-Ponty: Une ontologie de l’imaginaire", en: Phaenomenologica, v. CCIV, Springer. https://doi.org/10.1007/978-94-007-1975-0

Freud, S., "Tres ensayos de teoría sexual y otras obras", en: Obras Completas, v. VII, Etcheverry, J. (trad.), Buenos Aires: Amorrortu Editores, 1983, pp. 109-211. Traducido de: Drei Abhandlungen zur Sexualtheorie, Leipzig y Viena, Deuticke, 6ta. ed., 1925.

Kearney, R., "Ecrire la chair: L'expression diacritique chez Merleau-Ponty", en: Chiasmi International, v. XV, 2014.

Kristensen, S., "Foi perceptive et foi expressive”, en: Chiasmi International, v. V, 2003. https://doi.org/10.5840/chiasmi2003537

Kristensen, S., Parole et subjectivité. Merleau-Ponty et la phénoménologie de l'expression, Hildesheim: Georg Olms Verlag, 2010.

Merleau-Ponty, M., La structure du comportement, Paris: PUF, 1942.

Merleau-Ponty, M., Phénoménologie de la perception, Paris: Gallimard, 1945.

Merleau-Ponty, M., Signes, Paris: Gallimard, 1960.

Merleau-Ponty, M., Le visible et l'invisible, Paris: Gallimard, 1964.

Merleau-Ponty, M., Résumés de cours. Collège de France 1952-1960, Paris: Gallimard, 1968.

Merleau-Ponty, M., La prose du monde, Paris: Gallimard, 1969.

${ }^{88}$ Merleau-Ponty, M., Causeries, París: Éditions du Seuil, 2002. p. 59. 
Merleau-Ponty, M., Le primat de la perception et ses conséquences philosophiques, Grenoble, Cynara, 1989.

Merleau-Ponty, M., Parcours deux 1951-1961, Lonrai: Verdier, 2000.

Merleau-Ponty, M., Causeries, Paris: Éditions du Seuil, 2002.

Merleau-Ponty, M., L'institution dans l'histoire personnelle et publique. Le problème de la passivité, le sommeil, l'inconscient, la mémoire. Notes de Cours au Collège de France 1954-1955. París: Belin, 2003.

Merleau-Ponty, M., Le monde sensible et le monde de l'expression. Cours au Collège de France. Notes 1953. Ginebra: MêtisPresses, 2011.

Ralón de Walton, G., "La reversibilidad del silencio y el lenguaje”, en: Agora, v. XV, 1 (1996). 